\title{
RESOLUÇÕES ALTERNATIVAS PARA ALGUNS PROBLEMAS DE INDUÇÃO FINITA
}

Luiz Henrique de Lima Corrêa, Antonio Carlos Tamarrozzi

Universidade Federal de Mato Grosso do Sul. Grupo PET Matematica, Matemática-Três Lagoas

luizhenrique.napoli@gmail.com.

Agência de fomento: FNDE

\section{RESUMO}

O método de Indução Finita é uma técnica de demonstração de proposições matemáticas utilizado para validar diversos resultados no universo dos números naturais. Neste trabalho procuramos mostrar que algumas resoluções de problemas de Indução Finita podem ter resoluções alternativas e não convencionais. Muitas vezes a resolução intuitiva pode ser substituída por uma resolução mais complexa, que, no entanto, evidencia técnicas interessantes da Matemática não observados nas técnicas tradicionais de indução. Exemplos neste sentido, são casos de igualdades formadas por somas, onde os passos indutivos são aplicados na somatória e não na expressão condensada resultante.

Palavras-chave: Números Naturais, Técnicas de Demonstrações, Indução Finita )

\section{ALTERNATIVE RESOLUTIONS TO FINITE INDUCTION PROBLEMS}

\begin{abstract}
The Finite Induction method is a mathematical proposition demonstration technique used to validate several results in the universe of natural numbers. In this work we try to show that some resolutions of Finite Induction problems can have alternative and unconventional resolutions. Often intuitive resolution can be replaced by a more complex resolution, which nevertheless demonstrates interesting mathematical techniques not observed in traditional induction techniques. Examples in this sense are cases of equalities formed by sums, where the inductive steps are applied in the summation and not in the resulting condensed expression.

Keywords: (Principle of Finite Induction, Peano Axioms, Set of Natural Numbers)
\end{abstract}




\section{INTRODUÇÃO}

O principio de Indução Finta é um eficiente instrumento para a demonstração de diversas propriedades cujo domínio é o conjunto dos números naturais. Este método assegura a validade de uma propriedade $\mathrm{P}(n)$, para infinitos valores de $n \in \mathbb{N}$, com a verificação de duas condições:

i) $\exists n_{0} \in \mathbb{N}$ tal que $\mathrm{P}\left(n_{0}\right)$ é verdadeira.

ii) Todo $k \in \mathbb{N}, k \geq n_{0}$ satisfaz, $\mathrm{P}(k)$ verdadeira $\rightarrow \mathrm{P}(k+1)$ verdadeira.

Satisfeitas i) e ii), pode ser assegurado que $\mathrm{P}(n)$ é uma proposição verdadeira para todo $n \geq n_{0}$.

O principio de Indução Finta pode ser visto como consequência dos Axiomas de Peano. Giuseppe Peano (1858-1932) propôs uma lista de axiomas, que caracteriza o conjunto dos números naturais, baseado na noção de sucessor de um número natural:

i) Todo número natural tem um único sucessor, que também é um número natural.

ii) Números naturais diferentes têm sucessores diferentes.

iii) Existe um único número natural, designado por 1, que não é sucessor de nenhum outro.

iv) Seja $X$ um conjunto de números naturais. Se 1 pertence a $X$ e se, além disso, o sucessor de cada elemento de $X$ ainda pertence a $X$, então $X=\mathbb{N}$.

O processo de indução finita, lembra o comportamento das peças de um jogo de dominó.

1) A peça $n_{0}$ cai.

2) Se a peça $k$-enésima cai , a peça seguinte $(k+1)$ também cai.

Observamos que a execução destas duas etapas, asseguram que todas as peças cairão.

Este trabalho surgiu dos questionamentos da turma do primeiro ano do curso de Matemática da UFMS/ CPTL, diante de problemas de indução que envolvem somas. Por exemplo a famosa identidade relacionada a soma dos $n$ primeiros números naturais:

$$
1+2+3+4+\cdots+n=\frac{n(n+1)}{2}
$$

descoberta por Carl Friedrich Gauss (1777 - 1855). Quando se demonstra esta propriedade pelo método de indução finita, normalmente aplica-se o processo indutivo (passos i) e ii)), iniciando-se da esquerda para a direita, como vemos a seguir.

i. Para $n=1$, a igualdade é confirmada, visto que

$$
\frac{n(n+1)}{2}=\frac{1(1+1)}{2}=1=1+2+3+4+\cdots+n
$$

ii. Suponhamos verdadeira para $n=k$, ou seja

$$
1+2+3+4+\cdots+k=\frac{k(k+1)}{2},
$$

Observamos que aplicando a hipótese de indução $(*)$, temos

$$
1+2+3+\cdots+k+(k+1)=\frac{k(k+1)}{2}+(k+1)=\frac{k(k+1)+2(k+1)}{2}=\frac{(k+1)(k+2)}{2}
$$

o que assegura ser $\mathrm{P}(k+1)$ também é verdadeira.

Portanto a proposição é verdadeira para todo $n \geq 1$.

Surgiu então o impasse de questionar o que aconteceria se o processo indutivo se iniciasse a partir da expressão $\frac{n(n+1)}{2}$, obviamente a demonstração deveria ser concluída, tendo em vista a igualdade. Esta verificação por parte do grupo de alunos, deu inicio a um estudo interessante, que apresentamos neste trabalho.

\section{METODOLOGIA}

A Indução finita normalmente estudada nos cursos de matemática constitui uma técnica de demonstração amplamente eficiente para resolução de proposições referentes ao conjunto dos números naturais, sendo que esta técnica decorre dos axiomas de Peano, no século passado.

A inquietação dos alunos do primeiro ano do curso de Matemática durante os atendimentos de monitoria relacionados a este conceito, motivaram o grupo de alunos do PET 
(Programa de Educação Tutorial) a explorar situações alternativas para a demonstrações de resultados matemáticos com o processo de indução finita.

O desenvolvimento do trabalho foi obtido através da manipulação algébrica de conceitos matemáticos simples, usando técnicas de indução finita.

\section{RESULTADOS}

Vamos considerar novamente a "Soma de Gauss",

$$
1+2+3+4+\cdots+n=\frac{n(n+1)}{2}
$$

que motivou o surgimento deste trabalho. Aplicaremos o processo indutivo a partir da expressão $\frac{n(n+1)}{2}$, seria como considerarmos a igualdade como

$$
\frac{n(n+1)}{2}=1+2+3+4+\cdots+n \text {. }
$$

Temos:

i. Para $n=1$,

$$
\frac{n(n+1)}{2}=\frac{1(1+1)}{2}=1=1+2+3+4+\cdots+n
$$

ii. Suponhamos verdadeira para $n=k$, ou seja;

$$
\frac{k(k+1)}{2}=1+2+3+4+\cdots+k \text {. }
$$

E devemos provar que $k+1$ também é verdadeira, ou seja;

$$
\frac{(k+1)(k+2)}{2}=1+2+3+\cdots+k+(k+1) .
$$

Temos,

$$
\frac{(k+1)(k+2)}{2}=\frac{k(k+1)+2(k+1)}{2}=\frac{k(k+1)}{2}+(k+1)
$$

daí, aplicando a hipótese de indução $\left({ }^{*}\right)$ temos

$$
\frac{(k+1)(k+2)}{2}=\frac{k(k+1)}{2}+(k+1)=1+2+3+\cdots+k+(k+1),
$$

o que prova a afirmação para k+1. Portanto, a identidade de Gauss está verificada.

Mostraremos, na sequencias, outros exemplos, explorando esta técnica. O primeiro deles esta relacionada à expressão para a soma dos $n$ primeiros termos de uma progressão geométrica (PG)

1) Verificar que para todo $n \in \mathbb{N}^{*}$, é válido que,

$$
1+a^{2}+\cdots+a^{n-1}=\frac{a^{n}-1}{a-1} .
$$

i. Testemos para $n=1$,

$$
\frac{a^{n}-1}{a-1}=\frac{a^{1}-1}{a-1}=1=1+a^{2}+\cdots+a^{n-1}
$$

ii. Suponhamos verdadeira para $n=k$, ou seja;

$$
\frac{a^{k}-1}{a-1}=1+a^{2}+\cdots+a^{k-1} .
$$

E devemos provar que $\mathrm{P}(k+1)$ também é verdadeiro, ou seja;

$$
\frac{a^{k+1}-1}{a-1}=1+a^{2}+\cdots+a^{k} \text {. }
$$

Temos,

$$
\frac{a^{k+1}-1}{a-1}=\frac{a^{k+1}-1+a^{k}-a^{k}}{a-1}=\frac{a^{k}-1+a^{k}(a-1)}{a-1}=\frac{a^{k}-1}{a-1}+a^{k},
$$

Daí, aplicando a hipótese de indução $\left(^{*}\right)$, temos 


$$
\frac{a^{k+1}-1}{a-1}=\frac{a^{k}-1}{a-1}+a^{k}=1+a^{2}+\cdots+a^{k-1}+a^{k}
$$

O que prova a afirmação para $k+1$.

Provando que $P(k+1)$ também é verdadeira.

Portanto a proposição é verdadeira para todo $n \geq 1$.

2) Verificar que para todo $n \in \mathbb{N}^{*}$, é válido que,

$$
1^{2}+2^{2}+\cdots+n^{2}=\frac{n(n+1)(2 n+1)}{6}, \text { para } n \geq 1 .
$$

i. Testamos para $n=1$,

$$
\frac{\mathrm{n}(\mathrm{n}+1)(2 \mathrm{n}+1)}{6}=\frac{1(1+1)(2 \times 1+1)}{6}=1=1^{2}+2^{2}+\cdots+n^{2}
$$

ii. Suponhamos verdadeira para $n=k$, ou seja;

$$
\frac{\mathrm{k}(\mathrm{k}+1)(2 \mathrm{k}+1)}{6}=1^{2}+2^{2}+\cdots+\mathrm{k}^{2}
$$

E devemos provar que $\mathrm{P}(k+1)$ também é verdadeiro, ou seja;

$$
\frac{(k+1)(k+2)(2 k+3)}{6}=1^{2}+2^{2}+\cdots+k^{2}+(k+1)^{2} \text {. }
$$

Temos,

$$
\begin{aligned}
& \frac{(k+1)(k+2)(2 k+3)}{6}=\frac{(k+1)}{6} \times(k+2)(2 k+3)= \\
& =\frac{(k+1)}{6} \times[k(2 k+3)+2(2 k+3)]=\frac{(k+1)}{6} \times\left[2 k^{2}+3 k+4 k+6\right]= \\
& =\frac{(k+1)}{6} \times\left[6 k+6+2 k^{2}+k\right]=\frac{(k+1)}{6} \times[6(k+1)+k(2 k+1)]= \\
& =\frac{6(k+1)^{2}+k(k+1)(2 k+1)}{6}=\frac{6(k+1)^{2}}{6}+\frac{k(k+1)(2 k+1)}{6}= \\
& =(k+1)^{2}+\frac{k(k+1)(2 k+1)}{6} .
\end{aligned}
$$

Daí, aplicando a hipótese de indução $(*)$, temos,

$$
\begin{aligned}
& \frac{(k+1)(k+2)(2 K+3)}{6}=\frac{k(k+1)(2 k+1)}{6}+(k+1)^{2}= \\
& =1^{2}+2^{2}+\cdots+k^{2}+(k+1)^{2} .
\end{aligned}
$$

O que prova a afirmação para $k+1$.

Provando que $\mathrm{P}(k+1)$ também é verdadeira.

Portanto a proposição é verdadeira para todo $n \geq 1$.

3) Verificar que para todo $n \in \mathbb{N}^{*}$, é válido que,

$$
\left(1-\frac{1}{2}\right)\left(1-\frac{1}{3}\right)\left(1-\frac{1}{4}\right) \ldots\left(1-\frac{1}{n}\right)=\frac{1}{n}, \text { para } n \geq 2
$$

i. Testemos para $n=2$,

$$
\frac{1}{n}=\frac{1}{2}=\left(1-\frac{1}{2}\right)\left(1-\frac{1}{3}\right) \ldots\left(1-\frac{1}{n}\right) .
$$

ii. Suponhamos verdadeira para $n=k$, ou seja;

$$
\frac{1}{k}=\left(1-\frac{1}{2}\right)\left(1-\frac{1}{3}\right)\left(1-\frac{1}{4}\right) \ldots\left(1-\frac{1}{k}\right) .
$$

E devemos provar que para $k+1$ também é verdadeiro, ou seja;

Temos,

$$
\frac{1}{k+1}=\left(1-\frac{1}{2}\right)\left(1-\frac{1}{3}\right) \ldots\left(1-\frac{1}{k}\right)\left(1-\frac{1}{k+1}\right) .
$$

$$
\begin{aligned}
& \frac{1}{k+1}=\frac{k}{k(k+1)}=\frac{(k+1)-1}{k(k+1)}=\frac{1}{k}-\frac{1}{k(k+1)}=\frac{1}{k}-\frac{1}{k} \times \frac{1}{k+1}= \\
& \frac{1}{k}\left(1-\frac{1}{k+1}\right) .
\end{aligned}
$$

Daí, aplicando a hipótese de indução (*), temos, 
$\frac{1}{k+1}=\frac{1}{k}\left(1-\frac{1}{k+1}\right)=\left(1-\frac{1}{2}\right)\left(1-\frac{1}{3}\right) \ldots\left(1-\frac{1}{k}\right)\left(1-\frac{1}{k+1}\right)$

O que prova a afirmação para $k+1$.

Provando que $\mathrm{P}(k+1)$ também é verdadeira.

Portanto a proposição é verdadeira para todo $n \geq 2$.

\section{DISCUSSÃO}

Observamos que algumas das demonstrações com indução finita mostraram-se bastante intuitivas e revelaram artifícios diferentes das resoluções convencionais. Tendo em vista este interesse, não estamos preocupados com o "pédagio" que estamos pagando ao obtermos demonstrações mais longas. Contudo, em alguns casos, o tratamento reverso, possibilita ganhos na resolução relacionados também ao tamanho da demonstração. Consideremos, por exemplo, a seguinte desigualdade

$$
n ! \geq 2^{n}, \text { para todo } n \in \mathbb{N}, n \geq 4
$$

i. Testemos para $n=4$,

$$
2^{n}<n ! \rightarrow 2^{4}<4 ! \rightarrow 16<24
$$

ii. Suponhamos verdadeira para $n=k$, ou seja;

$$
2^{k}<k \text { ! }
$$

E devemos provar que $\mathrm{P}(\mathrm{k}+1)$ também é verdadeira, ou seja;

$$
2^{k+1}<(k+1) \text { ! }
$$

Temos,

$$
2^{k} \cdot 2<(k+1) \cdot 2^{k}<(k+1) \cdot k !=(k+1) !
$$

Provando que $\mathrm{P}(k+1)$ também é verdadeira.

Portanto a proposição é verdadeira para todo $n \geq 4$.

\section{CONCLUSÃO}

Neste trabalho foram exploradas igualdades e desigualdades matemáticas que evidenciam como o processo de indução finita pode apresentar tratamentos matemáticos distintos quando se considera as hipóteses de indução iniciadas em lados diferentes da identidade ou desigualdade a ser provada.

Em alguns casos a resolução intuitiva pode ser substituída por uma resolução mais complexa, que, no entanto, evidencia técnicas interessantes da Matemática não observados nas técnicas tradicionais, ou intuitivas, da indução.

\section{REFERÊNCIAS}

DOMINGUES, HYGINO H.; IEZZI, G. Álgebra Moderna, São Paulo, Atual Editora LTDA, 1995.

DOMINGUES, HYGINO H.; IEZZI, G. Fundamentos de aritmética, São Paulo, Atual Editora LTDA, 1999.

GUIDORIZZI, HAMILTON, Um curso de cálculo, vol 1, São Paulo. Editora LTC, 1990. 